\title{
ESTIMATION OF ANISOTROPIC ELASTIC PROPERTIES OF CARBON FIBERS USING NANOINDENTATION
}

\author{
Thimmappa Shetty Guruprasad*, Vincent Keryvin, Alain Bourmaud
}

\author{
University of Bretagne Sud, UMR CNRS 6027, IRDL, 27 rue Armand Guillemot BP 92116, 56321 Lorient, \\ France \\ * corresponding author: thimmappa-shetty.guruprasad@univ-ubs.fr
}

\begin{abstract}
Understanding the mechanical behavior of carbon fiber reinforced polymers requires knowledge on the deformation behavior of carbon fibers, they are highly anisotropic and heterogeneous. Nanoindentation is an efficient method for determining the mechanical properties in small volumes of materials. For isotropic materials, a single nanoindentation test can evaluate an elastic properties of the material. But for anisotropic material, the difficulty increases since measured indentation modulus depends on five elastic parameters $\left(E_{l}, E_{t}, G_{l t}, \nu_{l t}\right.$, and $\left.\nu_{t t}\right)$ of the material. Nanoindentation experiments are performed on carbon fibers orientated between $0^{\circ}$ to $90^{\circ}$ at ten different orientations to the fiber axis. From theoretical models given by Vlassak et al. and Delafargue and Ulm, the elastic constants are predicted numerically by comparing the results of indentation modulus versus orientation angle with the experiments.
\end{abstract}

KEYWORDS: Anisotropy, carbon fibers, nanoindentation.

\section{INTRODUCTION}

Carbon fiber composites are an important class of materials, which have high tensile strength, high modulus, and lower weight. Due to its advantages, the composites are used in many structural applications such as in the aerospace, automotive and naval industries. For the better design of composites having good structural strength, knowing the elastic properties of individual carbon fibers and also the knowledge of predicting its mechanical response is essential. The carbon fibers are generally categorized into four different types based on its strength, namely intermediate modulus (IM), high modulus (HM), ultra-high modulus (UHM) and high resistance (HR). The mechanical strength of the composite material reinforced with unidirectional carbon fibers behave to be highly anisotropic in its fiber direction and the strength in the transverse direction is much weaker as compared to longitudinal direction [1].

The most notable experimental technique to determine the mechanical properties of small and delicate structures is through nanoindentation. The nanoindentation is generally used for measuring mechanical properties such as elastic modulus and hardness. Since the carbon fibers are highly anisotropic, determining the modulus in any one direction will not determine the complete set of elastic properties of the material. Assuming carbon fibers as transversely isotropic materials and due to symmetry we need to determine five elastic constants. So, the indentation modulus is a function of five elastic parameters of the material which are three modulus $\left(E_{l}, E_{t}, G_{l t}\right)$ and two Poisson's ratios $\left(\nu_{l t}, \nu_{t t}\right)$. Due to less number of available theoretical models and also due to difficulty in implementing them numerically, determining elastic constants is a challenging task.

Similar to carbon fibers, there exists an anisotropy in natural fibers, for e.g. Jäger et al. 2] determined the anisotropy in the elastic properties of wood cell walls using the nanoindentation technique. They predicted the elastic constants from the numerical solutions of Vlassak et al. 3]. Recently, Csanádi et al. [4] studied the anisotropy in the carbon fibers from a ceramic based carbon fiber composites, here the elastic constants were predicted using the same numerical solutions of Vlassak et al., but the experiments were done only on two independent directions i.e. at longitudinal and transverse directions. More recently, Duan et al. [5] determined the transverse and the shear modulus of three different types of carbon fibers. The fiber surfaces are prepared by focused ion beam technique and both AFM as well nanoidentation tests are performed to determine the indentation modulus. The results from the AFM are considered to determine the elastic constants using Swanson's model [6].

In this work, we will determine the elastic constants of carbon fibers of type IM2C using nanoindentation, they are PAN-based carbon fibers. First, nanoindentation experiments are performed to determine the anisotropic response by cutting the carbon fibers at varying orientation angles concerning to the axis of the fiber. The fibers are cut between $0^{\circ}$ (longitudinal direction) to $90^{\circ}$ (transverse direction) in steps of $10^{\circ}$. The conventional machining techniques are used to obtain the desired surface for the tests. The indentation modulus is calculated using a standard Oliver-Pharr method. Then, we determined the elastic constants numerically using theoretical models; one of them was proposed by Vlassak et al. [3. The model relates the elastic constants with the indentation modulus for the 
anisotropic materials. The other theoretical model is given by Delafargue and Ulm [7, which can predict the values only at $0^{\circ}$ and $90^{\circ}$.

\section{EXPERIMENTS}

\subsection{MATERIALS}

The epoxy-based carbon fiber composite material is chosen to investigate the elastic properties. The composite consists of polyacrylonitrile (PAN) type carbon fibers type IM2C (Hexcel Corporation), and it belongs to the category of intermediate modulus. The average diameter of the carbon fibers are measured to be $5.2 \mu \mathrm{m}$. To perform nanoindentation, samples are prepared by cutting the composite at orientation angles between $0^{\circ}$ (longitudinal direction) to $90^{\circ}$ (transverse direction) in steps of $10^{\circ}$ with respect to the axis of the carbon fibers. Then the composite samples are hot mounted in epoxy with orientation angle parallel to the cutting plane. The desired surface of the carbon fibers for the nanoindentation test is achieved by polishing the samples initially with sandpapers of 1000, 2000 and 4000 grit and then with diamond suspension having a particle size of $1 \mu \mathrm{m}$ and finally with colloidal silica particles of size $30 \mathrm{~nm}$.

\subsection{MicRoscopic ANALYSIS}

As carbon fibers are oriented between $0^{\circ}$ and $90^{\circ}$ in steps of $10^{\circ}$ concerning to the carbon fiber axis, the actual orientation may be different than the cutting angle due to the use of conventional cutting and polishing methods. To measure the actual orientation angle $(\theta)$, samples are analysed using the scanning electron microscopy (SEM, JEOL Ltd.), the angle is determined by measuring the size and shape of the carbon fibers from the SEM images. For $0^{\circ}$, the shape is a circle, for $90^{\circ}$ it is a rectangle as it is perpendicular to the axis and for rest of the angles it is a ellipse. Figure 1(a) and (b) shows the SEM images of the carbon fibers with orientation angles of $0^{\circ}$ and $70^{\circ}$ respectively. To measure the actual orientation angle of the carbon fiber, Image J software is used, where the SEM images of the carbon fibers are digitized and the dimensions of the cross section are measured. For $0^{\circ}-80^{\circ}$, the major and minor axes of the ellipse is measured from their respective SEM images, then the actual orientation angle is determined using the relation $\theta=\cos ^{-1}\left(\frac{b}{a}\right)$, where $a$ and $b$ are the major and minor axes of the ellipse. Figure 1 (c) shows the schematic of the ellipse and the method for calculating the actual orientation angle from the carbon fibers cross section.

\subsection{NANOINDENTATION}

Nanoindentation is an efficient method for determining the local mechanical response in small volume of materials. To determine the anisotopic elastic properties of the carbon fibers, indentations are performed perpendicular to its cross section which are having a

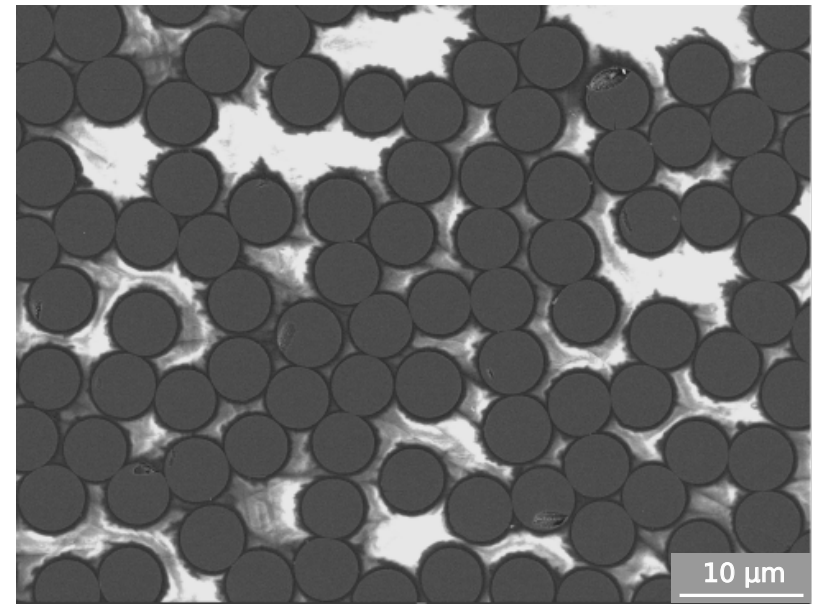

(a)

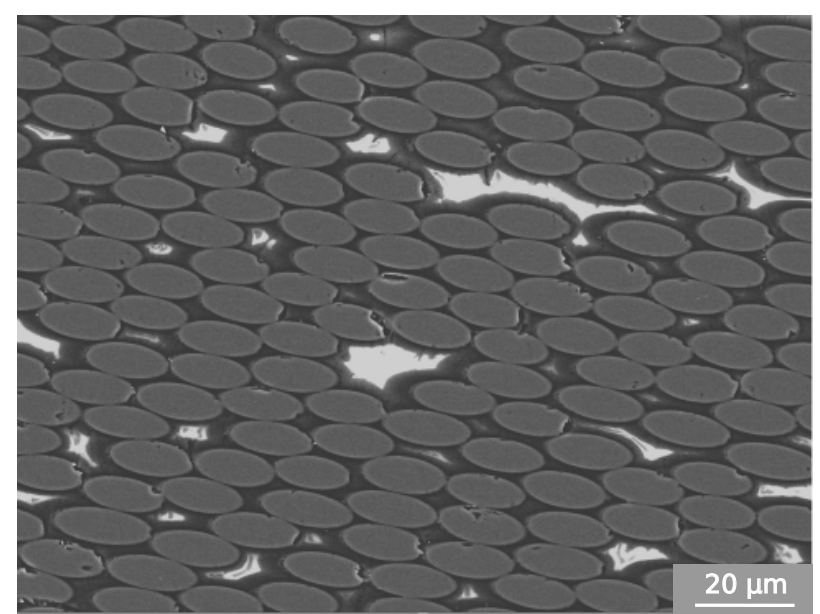

(b)

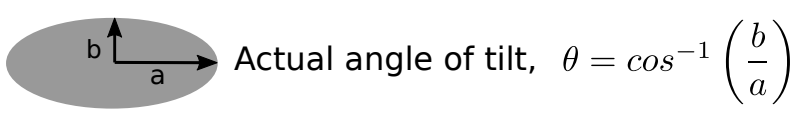

(c)

FIGURE 1. SEM images of Carbon fibers with cutting angle (a) $0^{\circ}$ and (b) $70^{\circ}$. A schematic with dimensions of ellipse and the formula to calculate the actual angle of tilt.

orientation angle varying from $0^{\circ}$ to $90^{\circ}$ with respect to the axis of the carbon fibers. Figure 2 shows the schematic of the indentation performed at an orientation angle $(\theta)$ with respect to the axis of the carbon fiber. The nanoindentation experiments are conducted in a Continuous stiffness measurement mode using an MTS Nano XP Nanoindenter having a diamond Berkovich tip. All the indentations are performed for depths less than $150 \mathrm{~nm}$, the larger depths the may affect the surrounding matrix along with the deformation of the carbon fibers. The hardness and elastic modulus are calculated using a standard method given by Oliver and Pharr (see 8 for detailed procedure for calculating the modulus and hardness). The effective 


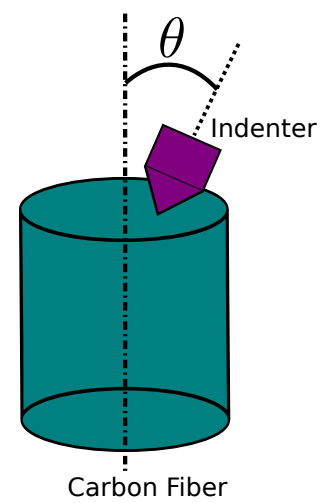

Figure 2. A schematic of the carbon fiber indentation at an orientation angle $(\theta)$ with respect to the carbon fiber axis.

indentation modulus is given by

$$
\frac{1}{M_{e f f}}=\frac{1-\nu_{i}^{2}}{E_{i}}+\frac{1}{M}
$$

where $M=\frac{E_{s}}{1-\nu_{s}^{2}}$, which is indentation modulus of the material. The $E_{i}$ and $E_{s}$ are the elastic modulus of the indenter and the sample respectively, similarly $\nu_{i}$ and $\nu_{s}$ are the respective Poisson ratios. The elastic modulus is calculated using the above relation, and the value of $\nu$ has a minor influence on the value of the elastic modulus 9. To determine the anisotropy in the carbon fibers, the samples prepared with varying orientation angle between $0^{\circ}$ to $90^{\circ}$ with respect to the axis of carbon fiber are indented. For each orientation angle, at least 20 are indents are performed to ensure the repeatability in the results.

\section{THEORETICAL MODELS FOR ANISOTROPIC NANOINDENTATION}

With the aim of determining the elastic properties of the material by nanoindentation, the indentation modulus for isotropic materials is given by

$$
M=\frac{E}{1-\nu^{2}},
$$

where $E$ is the elastic modulus of the material. For isotropic materials, $M$ is independent of the indentation direction.

In the case of anisotropic materials, the relation of indentation modulus with the elastic constants is very complicated as it depends on the indentation direction and the indenter shape.

The closed form expressions between the indentation modulus and the elastic material constants $\left(C_{i j}\right)$ for the transversely isotropic materials are proposed by Delafargue and Ulm [7] and the solution can be used when the indentation direction is aligned with direction of one of the principal material axes (see 7 for more details).

The solution of the indentation modulus is available only at two independent directions i.e. at longitudinal direction $0^{\circ}$ and transverse direction $90^{\circ}$. The indentation modulus in the longitudinal direction $\left(0^{\circ}\right)$ is given by

$$
M_{0^{\circ}}=2 \sqrt{\frac{C_{31}^{2}-C_{13}^{2}}{C_{11}}\left(\frac{1}{C_{44}}+\frac{2}{C_{31}+C_{13}}\right)^{-1}}
$$

where, $C_{31}=\sqrt{C_{11} C_{33}}$.

Similarly, the indentation modulus in the transverse direction $\left(90^{\circ}\right)$ is given as

$$
M_{90^{\circ}}=\sqrt{M_{12} M_{13}}
$$

where, $M_{12}=\sqrt{\frac{C_{11}}{C_{33}}} M_{3}$ and $M_{13}=\frac{C_{11}^{2}-C_{12}^{2}}{C_{11}}$.

The other well defined solution schemes for general anisotropic elastic materials are proposed by Vlassak et al. 3. The solution relates the indentation modulus with the elastic properties of the material as well as indentation direction.

The indentation modulus for the anisotropic material according to Vlassak et al. are given by

$$
M=\frac{1}{\alpha(e, \Phi)\left(1-e^{2}\right)^{\frac{1}{4}}}
$$

With the assumption of elliptical contact area formed with the indenter, the function $\alpha(e, \Phi)$ relates to eccentricity $(e)$ of the contact ellipse and angle between the major axis of the ellipse and the reference direction of a function $h$ (refer to 3 for further details).

The solutions of Vlassak et al. are used to determine the elastic constants by comparing the indentation modulus with the experimental results performed at varying orientation angles. The values of $E_{l}, E_{t}$, and $G_{l t}$ are varied such that the solution matches the experimental indentation modulus closely. The values of $\nu_{l t}$ and $\nu_{t t}$ are kept constant as their effect on the indentation modulus is considered to be negligible [2.

Similarly, the expressions of indentation modulus given by Delafargue and Ulm [7] are compared with the experiments to determine the elastic constants at two independent directions.

\section{Results AND DISCUSSIONS}

From microscopic analysis of the carbon fibers i.e., according to Section 2.2 we found that the actual angle of orientation is different than the cutting angle. For carbon fibers with $0^{\circ}$ i.e., carbon fibers orientated in a longitudinal direction, there exists small deviation in the orientation angle, and the actual angle was found to be $12^{\circ}$. But, for $90^{\circ}$ practical orientation remains same as the cutting angle. Also, it is found that there exists a small deviation in the orientation angle for the remaining cross-sectioned samples.

The results of nanoindentation experiments of carbon fibers with varying orientation angle with its axis are presented. The load $(\mathrm{P})$ versus the indentation 


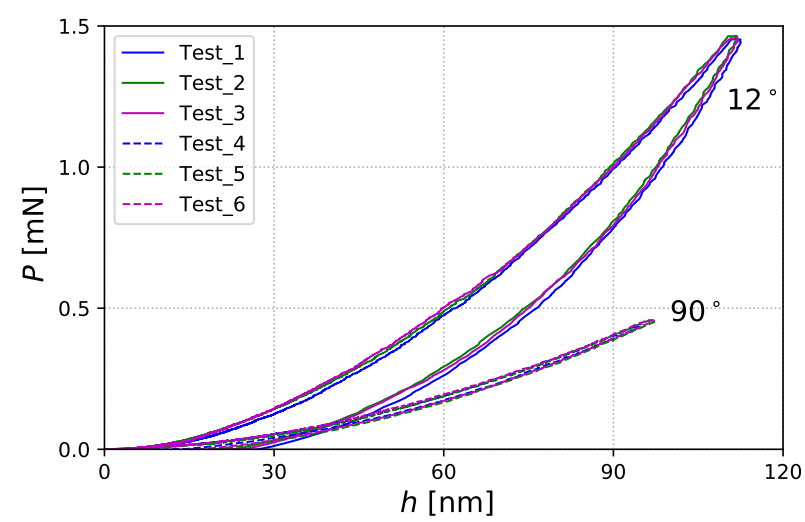

Figure 3. Load (P) versus indentation depth (h) behaviors from nanoidentation experiments for carbon fibers orientated at $12^{\circ}$ (solid lines) and $90^{\circ}$ (dotted lines).

depth (h) from the indentation experiments are plotted for all the samples between $12^{\circ}$ and $90^{\circ}$. As the diameters of the carbon fibers indented are $5.2 \mu \mathrm{m}$ and it is difficult to locate and perform indentation at the middle of the fibers. Figure 3 shows the representative $P-h$ curves of carbon fibers orientated at $12^{\circ}$ and $90^{\circ}$ with its axis. From the plot, we can see that there exists a repeatability in the $P-h$ curves for two of those orientations and similar repeatability in the $P-h$ are observed for other orientations as well. For the carbon fibers with orientation of $12^{\circ}$, we observe from the $P-h$ curves that there exists a hysteretic behavior and a small plastic deformation in the material but for the samples orientated at $90^{\circ}$ shows negligible plasticity and are nearly elastic. The $P-h$ also shows that the load required for the nearly longitudinal orientated fibers $\left(12^{\circ}\right)$ requires approximately three times the load in comparison to carbon fibers oriented in transverse direction $\left(90^{\circ}\right)$ to produce a same indentation depth.

The indentation modulus $(M)$ versus the actual orientation angle $(\theta)$ from the experiments are plotted in Figure 4 Due to anisotropy in the carbon fibers, the value of the indentation modulus decreases with increase in the orientation angle. Apart from the indentation modulus, we also observed a similar decreasing behavior in the hardness with increase in the orientation angle.

From the theoretical models presented in Section 3 elastic constants are determined from the nanoindentation experiments. Using Vlassak et al. solutions, the elastic constants such as $E_{l}, E_{t}$, and $G_{l t}$ are varied independently, such that a close match is obtained with the plot of experimental indentation modulus versus orientation angle. The final predicted values of three elastic constants are shown in Table 1 and the Poission's ratio's used are $\nu_{l t}=0.3, \nu_{t t}=0.3$. The corresponding fit with the experiments is shown in

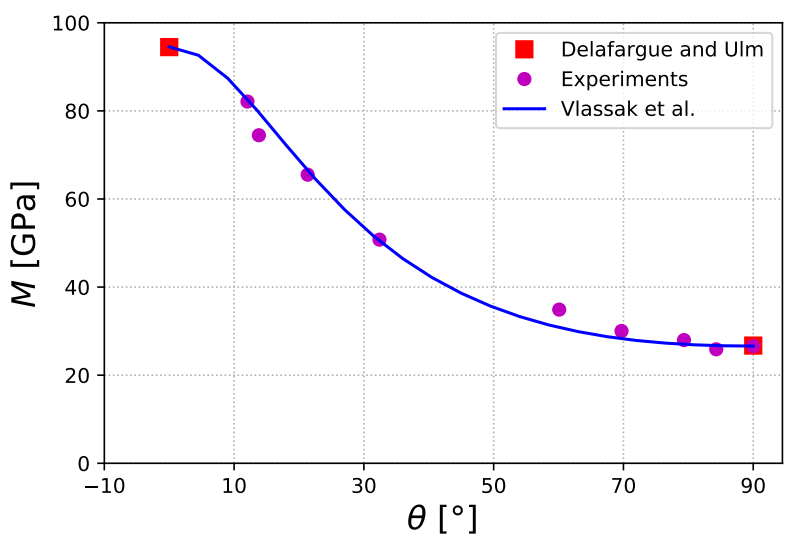

Figure 4. The plot of indentation modulus $(M)$ versus the orientation angle $(\theta)$ from nanoindentation experiments (circular markers), theoretical solutions of Delafargue and Ulm [7] (square markers) and Vlassak et al. 3] (solid line).

Figure 4 and the obtained fit has a good agreement with the nanoindentation experiments. Our predicted values of transverse modulus $\left(E_{t}\right)$ and the shear modulus $\left(G_{l t}\right)$ are comparable with other carbon fibers (type T-300), which was reported by Csanádi et al. [4] and the values are $E_{t}=30.9 \mathrm{GPa}, G_{l t}=10.9 \mathrm{GPa}$.

\begin{tabular}{ccc}
\hline$E_{l}(\mathrm{GPa})$ & $E_{t}(\mathrm{GPa})$ & $G_{l t}(\mathrm{GPa})$ \\
\hline 296 & 25.5 & 9 \\
\hline
\end{tabular}

TABLE 1. The predicted values of the elastic constants $\left(E_{l}, E_{t}\right.$, and $\left.G_{l t}\right)$ of carbon fibers (IM2C) via combined experimental-theoretical approach.

Similarly, the indentation modulus using the closedform solutions of Delafargue and Ulm are calculated for two orientations i.e., for $0^{\circ}$ and $90^{\circ}$ by varying the elastic constants. Here, the results shows a good agreement with Vlassak et al. as well as the indentation experiments.

\section{Conclusions}

The anisotropic elastic properties of carbon fibers (IM2C) are determined using combined experimentaltheoretical approach from nanoindentation. The carbon fibers are cut at varying orientation angles between $12^{\circ}$ and $90^{\circ}$, and they are indented to determine the indentation modulus between longitudinal orientation and the transverse orientations. Then, the plot of experimental indentation modulus versus the orientation angle is closely matched with the solutions of Delafargue and Ulm and also with the solutions of Vlassak et al. to predict the elastic constants. 


\section{LIST OF SYMBOLS}

$E_{l} \quad$ Elastic modulus in logitudinal direction [GPa]

$E_{t}$ Elastic modulus in transverse direction [GPa]

$G_{l t}$ Shear modulus [GPa]

$\nu_{l t}, \nu_{t t}$ Poisson's ratios

$M$ Indentation modulus [GPa]

$\theta$ Orientation angle $\left[{ }^{\circ}\right]$

$C_{i j} \quad$ Elastic constants of the material

$P$ Load $[\mathrm{mN}]$

$h \quad$ Indentation depth [nm]

\section{REFERENCES}

[1] R. Gibson. Principles of Composite Material Mechanics. Fourth edition. Boca Raton: CRC Press, 2016. DOI:https://doi.org/10.1201/b19626

[2] A. Jäger, T. Bader, K. Hofstetter, J. Eberhardsteiner. The relation between indentation modulus, microfibril angle, and elastic properties of wood cell walls.

Composites Part A: Applied Science and Manufacturing 42(6):677 - 685, 2011 DOI:https://doi.org/10.1016/j.compositesa.2011.02.007.

[3] J. Vlassak, M. Ciavarella, J. Barber, X. Wang. The indentation modulus of elastically anisotropic materials for indenters of arbitrary shape. Journal of the Mechanics and Physics of Solids 51(9):1701 - 1721, 2003. DOI:https://doi.org/10.1016/S0022-5096(03)00066-8

[4] T. Csanádi, D. Németh, C. Zhang, J. Dusza. Nanoindentation derived elastic constants of carbon fibres and their nanostructural based predictions.
Carbon 119:314 - 325, 2017.

DOI:https://doi.org/10.1016/j.carbon.2017.04.048

[5] S. Duan, F. Liu, T. Pettersson, et al. Determination of transverse and shear moduli of single carbon fibres. Carbon (158):772 - 782, 2020 DOI:https://doi.org/10.1016/j.carbon.2019.11.054

[6] S. R. Swanson. Hertzian contact of orthotropic materials. International Journal of Solids and Structures 41(7):1945 - 1959, 2004. DOI:https://doi.org/10.1016/j.ijsolstr.2003.11.003

[7] A. Delafargue, F.-J. Ulm. Explicit approximations of the indentation modulus of elastically orthotropic solids for conical indenters. International Journal of Solids and Structures 41(26):7351 - 7360, 2004. DOI:https://doi.org/10.1016/j.ijsolstr.2004.06.019

[8] W. Oliver, G. Pharr. An improved technique for determining hardness and elastic modulus using load and displacement sensing indentation experiments. Journal of Materials Research 7(6):1564-1583, 1992. DOI:10.1557/JMR.1992.1564

[9] W. Oliver, G. Pharr. Measurement of hardness and elastic modulus by instrumented indentation: Advances in understanding and refinements to methodology. Journal of Materials Research 19(1):3-20, 2004. DOI:10.1557/jmr.2004.19.1.3 